\title{
INVARIANT APPROXIMATIONS, GENERALIZED I-CONTRACTIONS, AND R-SUBWEAKLY COMMUTING MAPS
}

\author{
NASEER SHAHZAD
}

Received 11 May 2004 and in revised form 23 August 2004

We present common fixed point theory for generalized contractive $R$-subweakly commuting maps and obtain some results on invariant approximation.

\section{Introduction and preliminaries}

Let $S$ be a subset of a normed space $X=(X,\|\cdot\|)$ and $T$ and $I$ self-mappings of $X$. Then $T$ is called (1) nonexpansive on $S$ if $\|T x-T y\| \leq\|x-y\|$ for all $x, y \in S$; (2) Inonexpansive on $S$ if $\|T x-T y\| \leq\|I x-I y\|$ for all $x, y \in S$; (3) I-contraction on $S$ if there exists $k \in[0,1)$ such that $\|T x-T y\| \leq k\|I x-I y\|$ for all $x, y \in S$. The set of fixed points of $T$ (resp., $I$ ) is denoted by $F(T)$ (resp., $F(I)$ ). The set $S$ is called (4) $p$ starshaped with $p \in S$ if for all $x \in S$, the segment $[x, p]$ joining $x$ to $p$ is contained in $S$ (i.e., $k x+(1-k) p \in S$ for all $x \in S$ and all real $k$ with $0 \leq k \leq 1)$; (5) convex if $S$ is $p$ starshaped for all $p \in S$. The convex hull $\operatorname{co}(S)$ of $S$ is the smallest convex set in $X$ that contains $S$, and the closed convex hull $\operatorname{clco}(S)$ of $S$ is the closure of its convex hull. The mapping $T$ is called (6) compact if $\operatorname{cl} T(D)$ is compact for every bounded subset $D$ of $S$. The mappings $T$ and $I$ are said to be (7) commuting on $S$ if $I T x=T I x$ for all $x \in S$; (8) $R$-weakly commuting on $S$ [7] if there exists $R \in(0, \infty)$ such that $\|T I x-I T x\| \leq$ $R\|T x-I x\|$ for all $x \in S$. Suppose $S \subset X$ is $p$-starshaped with $p \in F(I)$ and is both $T$ - and $I$-invariant. Then $T$ and $I$ are called (8) $R$-subweakly commuting on $S$ [11] if there exists $R \in(0, \infty)$ such that $\|T I x-I T x\| \leq R \operatorname{dist}(I x,[T x, p])$ for all $x \in S$, where $\operatorname{dist}(I x,[T x, p])=\inf \{\|I x-z\|: z \in[T x, p]\}$. Clearly commutativity implies $R$-subweak commutativity, but the converse may not be true (see [11]).

The set $P_{S}(\hat{x})=\{y \in S:\|y-\hat{x}\|=\operatorname{dist}(\hat{x}, S)\}$ is called the set of best approximants to $\hat{x} \in X$ out of $S$, where $\operatorname{dist}(\hat{x}, S)=\inf \{\|y-\hat{x}\|: y \in S\}$. We define $C_{S}^{I}(\hat{x})=\{x \in S: I x \in$ $\left.P_{S}(\hat{x})\right\}$ and denote by $\mathfrak{I}_{0}$ the class of closed convex subsets of $X$ containing 0 . For $S \in \mathfrak{J}_{0}$, we define $S_{\hat{x}}=\{x \in S:\|x\| \leq 2\|\hat{x}\|\}$. It is clear that $P_{S}(\hat{x}) \subset S_{\hat{x}} \in \mathfrak{I}_{0}$.

In 1963, Meinardus [6] employed the Schauder fixed point theorem to establish the existence of invariant approximations. Afterwards, Brosowski [2] obtained the following extension of the Meinardus result. 
Theorem 1.1. Let $T$ be a linear and nonexpansive self-mapping of a normed space $X$, $S \subset X$ such that $T(S) \subset S$, and $\hat{x} \in F(T)$. If $P_{S}(\hat{x})$ is nonempty, compact, and convex, then $P_{S}(\hat{x}) \cap F(T) \neq \varnothing$.

Singh [15] observed that Theorem 1.1 is still true if the linearity of $T$ is dropped and $P_{S}(\hat{x})$ is only starshaped. He further remarked, in [16], that Brosowski's theorem remains valid if $T$ is nonexpansive only on $P_{S}(\hat{x}) \cup\{\hat{x}\}$. Then Hicks and Humphries [5] improved Singh's result by weakening the assumption $T(S) \subset S$ to $T(\partial S) \subset S$; here $\partial S$ denotes the boundary of $S$.

On the other hand, Subrahmanyam [18] generalized the Meinardus result as follows.

Theorem 1.2. Let $T$ be a nonexpansive self-mapping of $X, S$ a finite-dimensional $T$-invariant subspace of $X$, and $\hat{x} \in F(T)$. Then $P_{S}(\hat{x}) \cap F(T) \neq \varnothing$.

In 1981, Smoluk [17] noted that the finite dimensionality of $S$ in Theorem 1.2 can be replaced by the linearity and compactness of T. Subsequently, Habiniak [4] observed that the linearity of $T$ in Smoluk's result is superfluous.

In 1988, Sahab et al. [8] established the following result which contains Singh's result as a special case.

THeOREM 1.3. Let $T$ and I be self-mappings of a normed space $X, S \subset X$ such that $T(\partial S) \subset$ $S$, and $\hat{x} \in F(T) \cap F(I)$. Suppose $T$ is I-nonexpansive on $P_{S}(\hat{x}) \cup\{\hat{x}\}, I$ is linear and continuous on $P_{S}(\hat{x})$, and $T$ and $I$ are commuting on $P_{S}(\hat{x})$. If $P_{S}(\hat{x})$ is nonempty, compact, and $p$-starshaped with $p \in F(I)$, and if $I\left(P_{S}(\hat{x})\right)=P_{S}(\hat{x})$, then $P_{S}(\hat{x}) \cap F(T) \cap F(I) \neq \varnothing$.

Recently, Al-Thagafi [1] generalized Theorem 1.3 and proved some results on invariant approximations for commuting mappings. More recently, with the introduction of noncommuting maps to this area, Shahzad $[9,10,11,12,13,14]$ further extended Al-Thagafi's results and obtained a number of results regarding best approximations. The purpose of this paper is to present common fixed point theory for generalized $I$-contraction and $R$ subweakly commuting maps. As applications, some invariant approximation results are also obtained. Our results extend, generalize, and complement those of Al-Thagafi [1], Brosowski [2], Dotson Jr. [3], Habiniak [4], Hicks and Humphries [5], Meinardus [6], Sahab et al. [8], Shahzad [9, 10, 11, 12], Singh [15, 16], Smoluk [17], and Subrahmanyam [18].

\section{Main results}

Theorem 2.1. Let $S$ be a closed subset of a metric space $(X, d)$, and $T$ and $I R$-weakly commuting self-mappings of $S$ such that $T(S) \subset I(S)$. Suppose there exists $k \in[0,1)$ such that

$$
d(T x, T y) \leq k \max \left\{d(I x, I y), d(I x, T x), d(I y, T y), \frac{1}{2}[d(I x, T y)+d(I y, T x)]\right\}
$$

for all $x, y \in S$. If $\mathrm{cl}(T(S))$ is complete and $T$ is continuous, then $S \cap F(T) \cap F(I)$ is singleton. 
Proof. Let $x_{0} \in S$ and let $x_{1} \in S$ be such that $I x_{1}=T x_{0}$. Inductively, choose $x_{n}$ so that $I x_{n}=T x_{n-1}$. This is possible since $T(S) \subset I(S)$. Notice

$$
\begin{aligned}
d\left(I x_{n+1}, I x_{n}\right)= & d\left(T x_{n}, T x_{n-1}\right) \\
\leq & k \max \left\{d\left(I x_{n}, I x_{n-1}\right), d\left(I x_{n}, T x_{n}\right), d\left(I x_{n-1}, T x_{n-1}\right),\right. \\
& \left.\frac{1}{2}\left[d\left(I x_{n}, T x_{n-1}\right)+d\left(I x_{n-1}, T x_{n}\right)\right]\right\} \\
= & k \max \left\{d\left(I x_{n}, I x_{n-1}\right), d\left(I x_{n}, T x_{n}\right),\right. \\
& \left.d\left(I x_{n-1}, T x_{n-1}\right), \frac{1}{2} d\left(I x_{n-1}, T x_{n}\right)\right\} \\
\leq & k \max \left\{d\left(I x_{n}, I x_{n-1}\right), d\left(I x_{n}, T x_{n}\right),\right. \\
& \left.\frac{1}{2}\left[d\left(I x_{n-1}, I x_{n}\right)+d\left(I x_{n}, T x_{n}\right)\right]\right\} \\
\leq & k d\left(I x_{n}, I x_{n-1}\right)
\end{aligned}
$$

for all $n$. This shows that $\left\{I x_{n}\right\}$ is a Cauchy sequence in $S$. Consequently, $\left\{T x_{n}\right\}$ is a Cauchy sequence. The completeness of $\mathrm{cl}(T(S))$ further implies that $T x_{n} \rightarrow y \in S$ and so $I x_{n} \rightarrow y$ as $n \rightarrow \infty$. Since $T$ and $I$ are $R$-weakly commuting, we have

$$
d\left(T I x_{n}, I T x_{n}\right) \leq R d\left(T x_{n}, I x_{n}\right) .
$$

This implies that $I T x_{n} \rightarrow T y$ as $n \rightarrow \infty$. Now

$$
\begin{aligned}
d\left(T x_{n}, T T x_{n}\right) \leq k \max & \left\{d\left(I x_{n}, I T x_{n}\right), d\left(I x_{n}, T x_{n}\right), d\left(I T x_{n}, T T x_{n}\right),\right. \\
& \left.\frac{1}{2}\left[d\left(I x_{n}, T T x_{n}\right)+d\left(I T x_{n}, T x_{n}\right)\right]\right\} .
\end{aligned}
$$

Taking the limit as $n \rightarrow \infty$, we obtain

$$
\begin{aligned}
d(y, T y) \leq k \max \{d(y, T y), d(y, y), d(T y, T y), \\
\left.\frac{1}{2}[d(y, T y)+d(T y, y)]\right\} \\
=k d(y, T y),
\end{aligned}
$$

which implies $y=T y$. Since $T(S) \subset I(S)$, we can choose $z \in S$ such that $y=T y=I z$. Since

$$
\begin{gathered}
d\left(T T x_{n}, T z\right) \leq k \max \left\{d\left(I T x_{n}, I z\right), d\left(I T x_{n}, T T x_{n}\right), d(I z, T z),\right. \\
\left.\frac{1}{2}\left[d\left(I T x_{n}, T z\right)+d\left(I z, T T x_{n}\right)\right]\right\},
\end{gathered}
$$


taking the limit as $n \rightarrow \infty$ yields

$$
d(T y, T z) \leq k d(T y, T z)
$$

This implies that $T y=T z$. Therefore, $y=T y=T z=I z$. Using the $R$-weak commutativity of $T$ and $I$, we obtain

$$
d(T y, I y)=d(T I z, I T z) \leq R d(T z, I z)=0 .
$$

Thus $y=T y=I y$. Clearly $y$ is a unique common fixed point of $T$ and $I$. Hence $S \cap F(T) \cap$ $F(I)$ is singleton.

THeOREM 2.2. Let $S$ be a closed subset of a normed space $X$, and $T$ and I continuous selfmappings of $S$ such that $T(S) \subset I(S)$. Suppose I is linear, $p \in F(I), S$ is $p$-starshaped, and $\mathrm{cl}(T(S))$ is compact. If $T$ and I are R-subweakly commuting and satisfy

$$
\begin{gathered}
\|T x-T y\| \leq \max \{\|I x-I y\|, \operatorname{dist}(I x,[T x, p]), \operatorname{dist}(I y,[T y, p]), \\
\left.\frac{1}{2}[\operatorname{dist}(I x,[T y, p])+\operatorname{dist}(I y,[T x, p])]\right\}
\end{gathered}
$$

for all $x, y \in S$, then $S \cap F(T) \cap F(I) \neq \varnothing$.

Proof. Choose a sequence $\left\{k_{n}\right\} \subset[0,1)$ such that $k_{n} \rightarrow 1$ as $n \rightarrow \infty$. Define, for each $n$, a map $T_{n}$ by $T_{n}(x)=k_{n} T x+\left(1-k_{n}\right) p$ for each $x \in S$. Then each $T_{n}$ is a self-mapping of $S$. Furthermore, $T_{n}(S) \subset I(S)$ for each $n$ since $I$ is linear and $T(S) \subset I(S)$. Now the linearity of $I$ and the $R$-subweak commutativity of $T$ and $I$ imply that

$$
\begin{aligned}
\left\|T_{n} I x-I T_{n} x\right\| & =k_{n}\|T I x-I T x\| \leq k_{n} R \operatorname{dist}(I x,[T x, p]) \\
& \leq k_{n} R\left\|T_{n} x-I x\right\|
\end{aligned}
$$

for all $x \in S$. This shows that $T_{n}$ and $I$ are $k_{n} R$-weakly commuting for each $n$. Also

$$
\begin{aligned}
&\left\|T_{n} x-T_{n} y\right\|= k_{n}\|T x-T y\| \\
& \leq k_{n} \max \{\|I x-I y\|, \operatorname{dist}(I x,[T x, p]), \operatorname{dist}(I y,[T y, p]), \\
&\left.\frac{1}{2}[\operatorname{dist}(I x,[T y, p])+\operatorname{dist}(I y,[T x, p])]\right\} \\
& \leq k_{n} \max \left\{\|I x-I y\|,\left\|I x-T_{n} x\right\|,\left\|I y-T_{n} y\right\|,\right. \\
&\left.\frac{1}{2}\left[\left\|I x-T_{n} y\right\|+\left\|I y-T_{n} x\right\|\right]\right\}
\end{aligned}
$$

for all $x, y \in S$. Now Theorem 2.1 guarantees that $F\left(T_{n}\right) \cap F(I)=\left\{x_{n}\right\}$ for some $x_{n} \in S$. The compactness of $\operatorname{cl}(T(S))$ implies that there exists a subsequence $\left\{x_{m}\right\}$ of $\left\{x_{n}\right\}$ such 
that $x_{m} \rightarrow y \in S$ as $m \rightarrow \infty$. By the continuity of $T$ and $I$, we have $y \in F(T) \cap F(I)$. Hence $S \cap F(T) \cap F(I) \neq \varnothing$.

The following corollaries extend and generalize [3, Theorem 1] and [4, Theorem 4].

Corollary 2.3. Let $S$ be a closed subset of a normed space $X$, and $T$ and I continuous selfmappings of $S$ such that $T(S) \subset I(S)$. Suppose I is linear, $p \in F(I), S$ is p-starshaped, and $\mathrm{cl}(T(S))$ is compact. If $T$ and $I$ are $R$-subweakly commuting and $T$ is I-nonexpansive on $S$, then $S \cap F(T) \cap F(I) \neq \varnothing$.

Corollary 2.4. Let $S$ be a closed subset of a normed space $X$, and $T$ and $I$ continuous self-mappings of $S$ such that $T(S) \subset I(S)$. Suppose I is linear, $p \in F(I), S$ is p-starshaped, and $\operatorname{cl}(T(S))$ is compact. If $T$ and I are commuting and satisfy (2.9) for all $x, y \in S$, then $S \cap F(T) \cap F(I) \neq \varnothing$.

Let $D_{S}^{R, I}(\hat{x})=P_{S}(\hat{x}) \cap G_{S}^{R, I}(\hat{x})$, where

$$
G_{S}^{R, I}(\hat{x})=\{x \in S:\|I x-\hat{x}\| \leq(2 R+1) \operatorname{dist}(\hat{x}, S)\} .
$$

THeOrem 2.5. Let $T$ and I be self-mappings of a normed space $X$ with $\hat{x} \in F(T) \cap F(I)$ and $S \subset X$ such that $T(\partial S \cap S) \subset S$. Suppose I is linear on $D_{S}^{R, I}(\hat{x}), p \in F(I), D_{S}^{R, I}(\hat{x})$ is closed and $p$-starshaped, $\operatorname{cl} T\left(D_{S}^{R, I}(\hat{x})\right)$ is compact, and $I\left(D_{S}^{R, I}(\hat{x})\right)=D_{S}^{R, I}(\hat{x})$. If T and I are $R$ subweakly commuting and continuous on $D_{S}^{R, I}(\hat{x})$ and satisfy, for all $x \in D_{S}^{R, I}(\hat{x}) \cup\{\hat{x}\}$,

$$
\|T x-T y\| \leq\left\{\begin{array}{cl}
\|I x-I \hat{x}\| & \text { if } y=\hat{x}, \\
\max \{\|I x-I y\|, \operatorname{dist}(I x,[T x, p]), \operatorname{dist}(I y,[T y, p]), & \\
\left.\frac{1}{2}[\operatorname{dist}(I x,[T y, p])+\operatorname{dist}(I y,[T x, p])]\right\} & \text { if } y \in D_{S}^{R, I}(\hat{x}),
\end{array}\right.
$$

then $P_{S}(\hat{x}) \cap F(T) \cap F(I) \neq \varnothing$.

Proof. Let $x \in D_{S}^{R, I}(\hat{x})$. Then $x \in \partial S \cap S$ (see [1]) and so $T x \in S$ since $T(\partial S \cap S) \subset S$. Now

$$
\|T x-\hat{x}\|=\|T x-T \hat{x}\| \leq\|I x-I \hat{x}\|=\|I x-\hat{x}\|=\operatorname{dist}(\hat{x}, S) .
$$

This shows that $T x \in P_{S}(\hat{x})$. From the $R$-subweak commutativity of $T$ and $I$, it follows that

$$
\|I T x-\hat{x}\|=\|I T x-T \hat{x}\| \leq R\|T x-I x\|+\left\|I^{2} x-I \hat{x}\right\| \leq(2 R+1) \operatorname{dist}(\hat{x}, S) .
$$

This implies that $T x \in G_{S}^{R, I}(\hat{x})$. Consequently, $T x \in D_{S}^{R, I}(\hat{x})$ and $\operatorname{so} T\left(D_{S}^{R, I}(\hat{x})\right) \subset D_{S}^{R, I}(\hat{x})=$ $I\left(D_{S}^{R, I}(\hat{x})\right)$. Now Theorem 2.2 guarantees that $P_{S}(\hat{x}) \cap F(T) \cap F(I) \neq \varnothing$.

Theorem 2.6. Let $T$ and $I$ be self-mappings of a normed space $X$ with $\hat{x} \in F(T) \cap F(I)$ and $S \subset X$ such that $T(\partial S \cap S) \subset I(S) \subset S$. Suppose $I$ is linear on $D_{S}^{R, I}(\hat{x}), p \in F(I), D_{S}^{R, I}(\hat{x})$ is closed and p-starshaped, $\operatorname{cl} T\left(D_{S}^{R, I}(\hat{x})\right)$ is compact, and $I\left(G_{S}^{R, I}(\hat{x})\right) \cap D_{S}^{R, I}(\hat{x}) \subset I\left(D_{S}^{R, I}(\hat{x})\right) \subset$ $D_{S}^{R, I}(\hat{x})$. If $T$ and I are R-subweakly commuting and continuous on $D_{S}^{R, I}(\hat{x})$ and satisfy, for all $x \in D_{S}^{R, I}(\hat{x}) \cup\{\hat{x}\},(2.13)$, then $P_{S}(\hat{x}) \cap F(T) \cap F(I) \neq \varnothing$. 
Proof. Let $x \in D_{S}^{R, I}(\hat{x})$. Then, as in Theorem 2.5, Tx $\in D_{S}^{R, I}(\hat{x})$, that is, $T\left(D_{S}^{R, I}(\hat{x})\right) \subset$ $D_{S}^{R, I}(\hat{x})$. Also $\|(1-k) x+k \hat{x}-\hat{x}\|<\operatorname{dist}(\hat{x}, S)$ for all $k \in(0,1)$. This implies that $x \in \partial S \cap S$ (see [1]) and so $T\left(D_{S}^{R, I}(\hat{x})\right) \subset T(\partial S \cap S) \subset I(S)$. Thus we can choose $y \in S$ such that $T x=$ $I y$. Since $I y=T x \in P_{S}(\hat{x})$, it follows that $y \in G_{S}^{R, I}(\hat{x})$. Consequently, $T\left(D_{S}^{R, I}(\hat{x})\right) \subset$ $I\left(G_{S}^{R, I}(\hat{x})\right) \subset P_{S}(\hat{x})$. Therefore, $T\left(D_{S}^{R, I}(\hat{x})\right) \subset I\left(G_{S}^{R, I}(\hat{x})\right) \cap D_{S}^{R, I}(\hat{x}) \subset I\left(D_{S}^{R, I}(\hat{x})\right) \subset D_{S}^{R, I}(\hat{x})$. Now Theorem 2.2 guarantees that $P_{S}(\hat{x}) \cap F(T) \cap F(I) \neq \varnothing$.

Remark 2.7. Theorems 2.5 and 2.6 remain valid when $D_{S}^{R, I}(\hat{x})=P_{S}(\hat{x})$. If $I\left(P_{S}(\hat{x})\right) \subset$ $P_{S}(\hat{x})$, then $P_{S}(\hat{x}) \subset C_{S}^{I}(\hat{x}) \subset G_{S}^{R, I}(\hat{x})$ (see [1]) and so $D_{S}^{R, I}(\hat{x})=P_{S}(\hat{x})$. Consequently, Theorem 2.5 contains Theorem 1.3 as a special case.

The following result includes [1, Theorem 4.1] and [4, Theorem 8]. It also contains the well-known results due to Smoluk [17] and Subrahmanyam [18].

Theorem 2.8. Let $T$ be a self-mapping of a normed space $X$ with $\hat{x} \in F(T)$ and $S \in \mathfrak{I}_{0}$ such that $T\left(S_{\hat{x}}\right) \subset S$. If $\mathrm{cl} T\left(S_{\hat{x}}\right)$ is compact and $T$ is continuous on $S_{\hat{x}}$ and satisfies for all $x \in S_{\hat{x}} \cup\{\hat{x}\}$

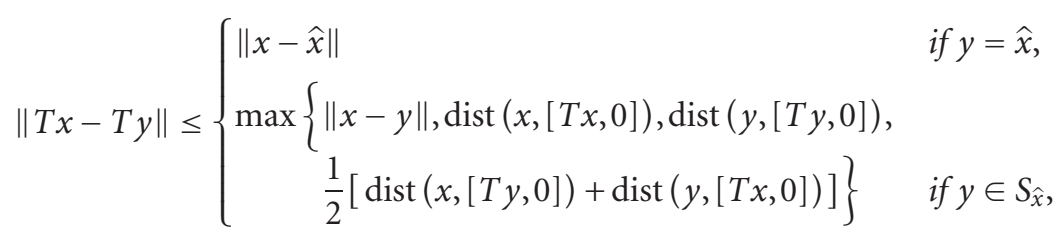

then

(i) $P_{S}(\hat{x})$ is nonempty, closed, and convex,

(ii) $T\left(P_{S}(\hat{x})\right) \subset P_{S}(\hat{x})$,

(iii) $P_{S}(\hat{x}) \cap F(T) \neq \varnothing$.

Proof. (i) We may assume that $\hat{x} \notin S$. If $x \in S \backslash S_{\hat{x}}$, then $\|x\|>2\|\hat{x}\|$. Notice that

$$
\|x-\hat{x}\| \geq\|x\|-\|\hat{x}\|>\|\hat{x}\| \geq \operatorname{dist}\left(\hat{x}, S_{\hat{x}}\right)
$$

Consequently, $\operatorname{dist}\left(\hat{x}, S_{\hat{x}}\right)=\operatorname{dist}(\hat{x}, S) \leq\|\hat{x}\|$. Also $\|z-\hat{x}\|=\operatorname{dist}\left(\hat{x}, \operatorname{cl} T\left(S_{\hat{x}}\right)\right)$ for some $z \in$ $\operatorname{cl} T\left(S_{\hat{x}}\right)$. Thus

$$
\begin{aligned}
\operatorname{dist}\left(\hat{x}, S_{\hat{x}}\right) & \leq \operatorname{dist}\left(\hat{x}, \operatorname{cl} T\left(S_{\hat{x}}\right)\right) \leq \operatorname{dist}\left(\hat{x}, T\left(S_{\hat{x}}\right)\right) \\
& \leq\|T x-\hat{x}\|=\|T x-T \hat{x}\| \\
& \leq\|x-\hat{x}\|
\end{aligned}
$$

for all $x \in S_{\hat{x}}$. This implies that $\|z-\hat{x}\|=\operatorname{dist}(\hat{x}, S)$ and so $P_{S}(\hat{x})$ is nonempty. Furthermore, it is closed and convex.

(ii) Let $y \in P_{S}(\hat{x})$. Then

$$
\|T y-\hat{x}\|=\|T y-T \hat{x}\| \leq\|y-\hat{x}\|=\operatorname{dist}(\hat{x}, S) .
$$

This implies that $T y \in P_{S}(\hat{x})$ and so $T\left(P_{S}(\hat{x})\right) \subset P_{S}(\hat{x})$. 
(iii) Theorem 2.2 guarantees that $P_{S}(\hat{x}) \cap F(T) \neq \varnothing$ since $\operatorname{cl} T\left(P_{S}(\hat{x})\right) \subset \operatorname{cl} T\left(S_{\hat{x}}\right)$ and $\operatorname{cl} T\left(S_{\hat{x}}\right)$ is compact.

TheOREM 2.9. Let $I$ and $T$ be self-mappings of a normed space $X$ with $\hat{x} \in F(I) \cap F(T)$ and $S \in \mathfrak{I}_{0}$ such that $T\left(S_{\hat{x}}\right) \subset I(S) \subset S$. Suppose that $I$ is linear, $\|I x-\hat{x}\|=\|x-\hat{x}\|$ for all $x \in S$, $\operatorname{cl} I\left(S_{\hat{x}}\right)$ is compact and I satisfies, for all $x, y \in S_{\hat{x}}$,

$$
\begin{array}{r}
\|I x-I y\| \leq \max \{\|x-y\|, \operatorname{dist}(x,[I x, 0]), \operatorname{dist}(y,[I y, 0]), \\
\left.\frac{1}{2}[\operatorname{dist}(x,[I y, 0])+\operatorname{dist}(y,[I x, 0])]\right\} .
\end{array}
$$

If I and T are R-subweakly commuting and continuous on $S_{\hat{x}}$ and satisfy, for all $x \in S_{\hat{x}} \cup\{\hat{x}\}$, and $p \in F(I)$,

$$
\|T x-T y\| \leq\left\{\begin{array}{cl}
\|I x-I \hat{x}\| & \text { if } y=\hat{x}, \\
\max \{\|I x-I y\|, \operatorname{dist}(I x,[T x, p]), \operatorname{dist}(I y,[T y, p]), & \\
\left.\frac{1}{2}[\operatorname{dist}(I x,[T y, p])+\operatorname{dist}(I y,[T x, p])]\right\} & \text { if } y \in S_{\hat{x},}
\end{array}\right.
$$

then

(i) $P_{S}(\hat{x})$ is nonempty, closed, and convex,

(ii) $T\left(P_{S}(\hat{x})\right) \subset I\left(P_{S}(\hat{x})\right) \subset P_{S}(\hat{x})$,

(iii) $P_{S}(\hat{x}) \cap F(I) \cap F(T) \neq \varnothing$.

Proof. From Theorem 2.8, (i) follows immediately. Also, we have $I\left(P_{S}(\hat{x})\right) \subset P_{S}(\hat{x})$. Let $y \in T\left(P_{S}(\hat{x})\right)$. Since $T\left(S_{\hat{x}}\right) \subset I(S)$ and $P_{S}(\hat{x}) \subset S_{\hat{x}}$, there exist $z \in P_{S}(\hat{x})$ and $x_{1} \in S$ such that $y=T z=I x_{1}$. Furthermore, we have

$$
\left\|I x_{1}-\hat{x}\right\|=\|T z-T \hat{x}\| \leq\|I z-I \hat{x}\| \leq\|z-\hat{x}\|=d(\hat{x}, S) .
$$

Thus $x_{1} \in C_{S}^{I}(\hat{x})=P_{S}(\hat{x})$ and so (ii) holds.

Since, by Theorem $2.8, P_{S}(\hat{x}) \cap F(I) \neq \varnothing$, it follows that there exists $p \in P_{S}(\hat{x})$ such that $p \in F(I)$. Hence (iii) follows from Theorem 2.2.

The following corollary extends [1, Theorem 4.2(a)] to a class of noncommuting maps. Corollary 2.10. Let $I$ and $T$ be self-mappings of a normed space $X$ with $\hat{x} \in F(I) \cap F(T)$ and $S \in \mathfrak{J}_{0}$ such that $T\left(S_{\hat{x}}\right) \subset I(S) \subset S$. Suppose that $I$ is linear, $\|I x-\hat{x}\|=\|x-\hat{x}\|$ for all $x \in S, \operatorname{cl} I\left(S_{\hat{x}}\right)$ is compact, and I is nonexpansive on $S_{\hat{x}}$. If I and T are $R$-subweakly commuting on $S_{\hat{x}}$ and $T$ is I-nonexpansive on $S_{\hat{x}} \cup\{\hat{x}\}$, then

(i) $P_{S}(\hat{x})$ is nonempty, closed and convex,

(ii) $T\left(P_{S}(\hat{x})\right) \subset I\left(P_{S}(\hat{x})\right) \subset P_{S}(\hat{x})$, and

(iii) $P_{S}(\hat{x}) \cap F(I) \cap F(T) \neq \varnothing$. 


\section{Acknowledgment}

The author would like to thank the referee for his suggestions.

\section{References}

[1] M. A. Al-Thagafi, Common fixed points and best approximation, J. Approx. Theory 85 (1996), no. 3, 318-323.

[2] B. Brosowski, Fixpunktsätze in der Approximationstheorie, Mathematica (Cluj) 11 (34) (1969), 195-220 (German).

[3] W. G. Dotson Jr., Fixed point theorems for non-expansive mappings on star-shaped subsets of Banach spaces, J. London Math. Soc. (2) 4 (1972), 408-410.

[4] L. Habiniak, Fixed point theorems and invariant approximations, J. Approx. Theory 56 (1989), no. 3, 241-244.

[5] T. L. Hicks and M. D. Humphries, A note on fixed-point theorems, J. Approx. Theory 34 (1982), no. 3, 221-225.

[6] G. Meinardus, Invarianz bei linearen Approximationen, Arch. Rational Mech. Anal. 14 (1963), 301-303 (German).

[7] R. P. Pant, Common fixed points of noncommuting mappings, J. Math. Anal. Appl. 188 (1994), no. 2, 436-440.

[8] S. A. Sahab, M. S. Khan, and S. Sessa, A result in best approximation theory, J. Approx. Theory 55 (1988), no. 3, 349-351.

[9] N. Shahzad, A result on best approximation, Tamkang J. Math. 29 (1998), no. 3, 223-226.

[10] Correction to: "A result on best approximation", Tamkang J. Math. 30 (1999), no. 2, 165.

[11] Invariant approximations and R-subweakly commuting maps, J. Math. Anal. Appl. 257 (2001), no. 1, 39-45.

[12] - Noncommuting maps and best approximations, Rad. Mat. 10 (2001), no. 1, 77-83.

[13] _ On R-subcommuting maps and best approximations in Banach spaces, Tamkang J. Math. 32 (2001), no. 1, 51-53.

[14] , Remarks on invariant approximations, Int. J. Math. Game Theory Algebra 13 (2003), no. 2, 157-159.

[15] S. P. Singh, An application of a fixed-point theorem to approximation theory, J. Approx. Theory 25 (1979), no. 1, 89-90.

[16] Application of fixed point theorems in approximation theory, Applied Nonlinear Analysis (Proc. Third Internat. Conf., Univ. Texas, Arlington, Tex, 1978) (V. Lakshmikantham, ed.), Academic Press, New York, 1979, pp. 389-394.

[17] A. Smoluk, Invariant approximations, Mat. Stos. (3) 17 (1981), 17-22 (Polish).

[18] P. V. Subrahmanyam, An application of a fixed point theorem to best approximation, J. Approximation Theory 20 (1977), no. 2, 165-172.

Naseer Shahzad: Department of Mathematics, King Abdul Aziz University, P.O. Box 80203, Jeddah 21589, Saudi Arabia

E-mail address: nshahzad@kaau.edu.sa 